\title{
ERRATUM
}

\section{Attenuation of neurological injury with early baicalein treatment following subarachnoid hemorrhage in rats}

TO THE EDITOR: We made a mistake while compiling figures for our article in the Journal of Neurosurgery (Kuo CP, Wen LL, Chen CM, et al: Attenuation of neurological injury with early baicalein treatment following subarachnoid hemorrhage in rats. J Neurosurg 119:10281037, 2013). We inadvertently included 2 photomicrographs of tissue samples that were previously published in another article by our group in Anesthesiology (Kuo CP, $\mathrm{Lu} \mathrm{CH}$, Wen LL, et al: Neuroprotective effect of curcumin in an experimental rat model of subarachnoid hemorrhage. Anesthesiology 115:1229-1238, 2011).

Specifically, Fig. 4A SAH group (tissue from the dentate gyrus [DG]) in our article in the Journal of Neurosurgery is the same photomicrograph as Fig. 4B (SAH group [tissue from DG]) in our article in Anesthesiology, and Fig. 4A SAH group (tissue from the cornu ammonis 3 [CA3]) in our article in the Journal of Neurosurgery is the same as Fig. 4J (SAH group [tissue from CA3]) in our article in Anesthesiology. The only difference between the matching panels is the placement of arrows over the photomicrographs.
Following identification of the duplicate figures in a Twitter post and the request for an explanation from the Journal of Neurosurgery, we carefully reviewed the 2 articles and found that, indeed, we were in error during submission of photomicrographs for our Journal of Neurosurgery paper.

Our intention was not to reproduce previously published photographs. We performed separate experiments for the 2 studies and had ample photomicrographs of tissue samples from both studies. Unfortunately, there was a mix-up in compiling figures for the second paper.

We sincerely apologize for our mistake. Please be assured that our error did not change the results or conclusions of our paper.

We thank the Editor for allowing us to replace the 2 panels in Fig. 4, as shown on the following page. The article has been corrected online as of July 14, 2017.

Zhi-Fu Wu, MD

Tri-Service General Hospital and National Defense Medical Center, Taipei, Taiwan, R.O.C.

\section{INCLUDE WHEN CITING}

Published online July 14, 2017; DOI: 10.3171/2017.6.JNS121919a.

@AANS, 2017 

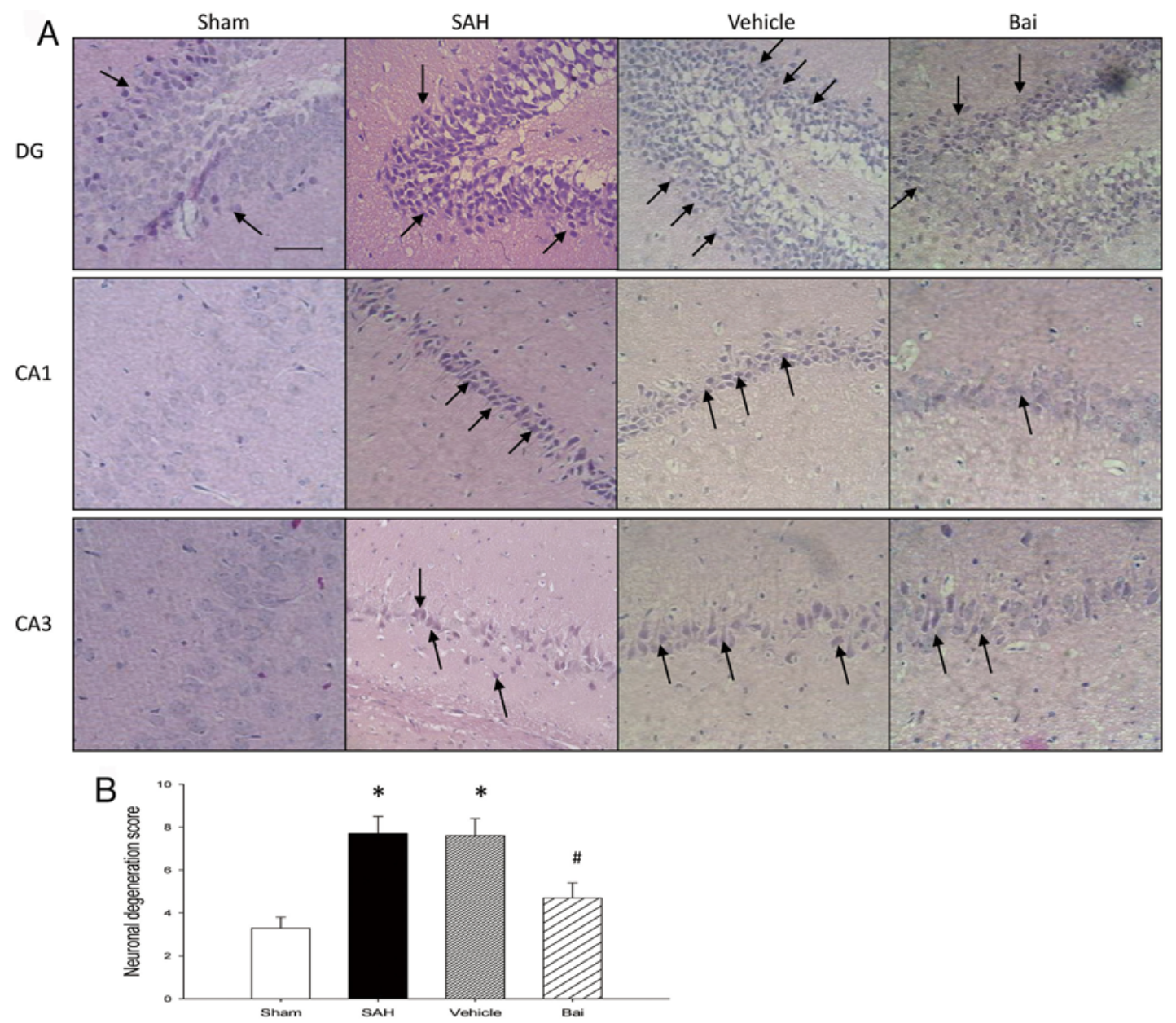

FIG. 4. A: The morphology of neurons in the hippocampus. Arrows indicate degenerated neurons, which were marked by the presence of pyknotic nuclei, dark cells, and vacuolar spaces. $\mathrm{H} \& \mathrm{E}$, bar $=50 \mu \mathrm{m}$. B: Neuronal degeneration was normal to mild and mild to moderate in the sham and baicalein rats. In the SAH and vehicle rats, the degree of neuronal degeneration was moderate to severe. Early treatment with baicalein significantly reduced neuronal degeneration $(n=6)$. ${ }^{*} p<0.05$, significant difference from sham and baicalein rats; $\# p<0.05$, significant difference from sham rats. Figure is available in color online only. 